\title{
Stabilizing Canonical-Ensemble Calculations in the Auxiliary-Field Monte Carlo Method
}

\author{
C. N. Gilbreth* and Y. Alhassid \\ Center for Theoretical Physics, Sloane Physics Laboratory, \\ Yale University, New Haven, CT 06520, USA
}

\begin{abstract}
Quantum Monte Carlo methods are powerful techniques for studying strongly interacting Fermi systems. However, implementing these methods on computers with finite-precision arithmetic requires careful attention to numerical stability. In the auxiliary-field Monte Carlo (AFMC) method, low-temperature or large-model-space calculations require numerically stabilized matrix multiplication. When adapting methods used in the grand-canonical ensemble to the canonical ensemble of fixed particle number, the numerical stabilization increases the number of required floating-point operations for computing observables by a factor of the size of the single-particle model space, and thus can greatly limit the systems that can be studied. We describe an improved method for stabilizing canonical-ensemble calculations in AFMC that exhibits better scaling, and present numerical tests that demonstrate the accuracy and improved performance of the method.
\end{abstract}

\footnotetext{
(C) 2014. This manuscript version is made available under the Elsevier user license http://www.elsevier.com/open-access/userlicense/1.0/

* cngilbreth@gmail.com

$\dagger$ yoram.alhassid@yale.edu
} 


\section{INTRODUCTION}

The auxiliary-field quantum Monte Carlo (AFMC) method is a widely used approach for calculating ground-state and finite-temperature properties of interacting quantum manyfermion systems. These include nuclei [1], condensed matter systems [2, 3], atoms and molecules [4, 5], quark matter [6], and cold atomic Fermi gases [7-10]. It is one of the few general numerical methods for such systems that takes into account all two-body correlations of the particles, and is exact up to a statistical error introduced by the Monte Carlo sampling. In general, fermionic systems can suffer from a so-called sign problem that leads to large statistical errors. However, for a significant class of systems, namely those with "good sign" interactions, the statistical errors are reasonable, and AFMC is one of the most robust and accurate techniques available.

Implementing AFMC at low temperatures and/or with large single-particle model spaces can be numerically challenging. In this method, the thermal propagator $\hat{U}=e^{-\beta \hat{H}}$, where $\hat{H}$ is the Hamiltonian of the system and $\beta=1 / T$ is the inverse temperature, is expressed as a functional integral over non-interacting propagators $\hat{U}(\sigma)$ parameterized by fields $\sigma$ that depend on imaginary time. The time dependence of these auxiliary fields requires that $\hat{U}(\sigma)$ be represented as a long chain of matrix multiplications, with each matrix representing the propagator for a short time slice $\Delta \beta$. At low temperatures (i.e., large $\beta$ ), or for large singleparticle spaces, the repeated matrix multiplications give rise to widely varying numerical scales, in which the intermediate and smaller scales are hidden in the differences between much larger numbers. In finite-precision arithmetic, these physically important intermediate and smaller scales become unrecoverable, leading to uncontrolled numerical errors.

In the grand-canonical ensemble, this problem has a well-known solution [3] in which the matrix representation of $\hat{U}(\sigma)$ in the single-particle model space is decomposed into a form that displays the scales explicitly. However, when this stabilization method is applied in a straightforward manner to the canonical ensemble [11], in which exact particle-number projection is used, it increases the computational effort required for computing observables from $O\left(N_{s}^{3}\right)$ to $O\left(N_{s}^{4}\right)$, thus limiting the sizes of systems which can be studied.

Here we describe an improved method for numerically stabilized AFMC calculations in the canonical ensemble. This method scales as $O\left(N_{s}^{3}\right)$ and therefore makes canonicalensemble calculations competitive with grand-canonical calculations. In practice it allows 


\section{AUXILIARY-FIELD MONTE CARLO (AFMC) METHOD}

The AFMC method employs a Hubbard-Stratonovich (HS) transformation [12, 13] to rewrite the thermal propagator $e^{-\beta \hat{H}}$ of a many-particle system as a functional integral of a non-interacting propagator $\hat{U}(\sigma)$ for particles moving in imaginary-time-dependent fields $\sigma$. Here $\hat{H}$ is the Hamiltonian, $\beta=1 / T$ is the inverse temperature, and the auxiliary fields $\sigma=\sigma(\tau)$ are functions of the imaginary time $\tau(0 \leq \tau \leq \beta)$. Explicitly,

$$
e^{-\beta \hat{H}}=\int D[\sigma] G(\sigma) \hat{U}(\sigma)
$$

where $D[\sigma]$ is the integration measure, $G(\sigma)$ is a Gaussian weight, and $\hat{U}(\sigma)$ is the thermal propagator for a non-interacting system parameterized by the auxiliary fields $\sigma(\tau)$.

The Hamiltonian $\hat{H}$ on the left-hand-side of Eq. (1) is defined in a many-particle fermionic Fock space. This is typically generated from a finite basis of $N_{s}$ single-particle orbitals by constructing all possible Slater determinants in which a subset of these orbitals are occupied. The resulting space has very large dimension, e.g., $\left(\begin{array}{c}N_{s} \\ N\end{array}\right)$ for $N$ fermions of a single species. On the other hand, the propagator $\hat{U}(\sigma)$ in the integrand of Eq. (1) describes a non-interacting system, and its properties can be determined by matrix algebra in the space of single-particle states, which has much lower dimension $N_{s}$. 
Observables in the AFMC method are computed by sampling their thermal expectation values in this noninteracting system at different values of the external fields $\sigma$. For an observable $\hat{O}$, the HS transformation implies

$$
\langle\hat{O}\rangle=\frac{\operatorname{Tr}\left(\hat{O} e^{-\beta \hat{H}}\right)}{\operatorname{Tr}\left(e^{-\beta \hat{H}}\right)}=\frac{\int D[\sigma] G(\sigma) \operatorname{Tr}[\hat{O} \hat{U}(\sigma)]}{\int D[\sigma] G(\sigma) \operatorname{Tr}[\hat{U}(\sigma)]} .
$$

The traces in Eq. (21) can be computed in various subspaces of Fock space, including the grand-canonical ensemble (where a suitable chemical potential must also be included), and the canonical ensemble. Traces at given values of good quantum numbers, such as spin and parity, can also be calculated by using suitable projection operators [14 16].

In the grand-canonical (GC) ensemble, the many-particle traces can be easily computed from the matrix representation $U(\sigma)$ of the propagator $\hat{U}(\sigma)$ in the single-particle space. The corresponding partition function is

$$
\zeta(\sigma)=\operatorname{Tr}_{\mathrm{GC}}[\hat{U}(\sigma)]=\operatorname{det}[1+U(\sigma)]
$$

while the expectation of a one-body operator $a_{i}^{\dagger} a_{j}$ is

$$
\left\langle a_{i}^{\dagger} a_{j}\right\rangle_{\sigma}=\frac{\operatorname{Tr}_{\mathrm{GC}}\left[a_{i}^{\dagger} a_{j} \hat{U}(\sigma)\right]}{\operatorname{Tr}_{\mathrm{GC}}[\hat{U}(\sigma)]}=\left[\frac{1}{1+U(\sigma)^{-1}}\right]_{j, i} .
$$

To compute these quantities in an $\mathrm{N}$-particle canonical ensemble, one can apply a discrete Fourier sum to grand-canonical quantities [17]. This yields [18]

$$
\zeta_{N}(\sigma)=\frac{1}{N_{s}} \sum_{m=1}^{N_{s}} e^{-i \varphi_{m} N} \operatorname{det}\left[1+e^{i \varphi_{m}} U(\sigma)\right]
$$

and

$$
\left\langle a_{i}^{\dagger} a_{j}\right\rangle_{N, \sigma}=\frac{1}{\zeta_{N}(\sigma) N_{s}} \sum_{m=1}^{N_{s}} e^{-i \varphi_{m} N}\left[\frac{1}{1+e^{-i \varphi_{m}} U(\sigma)^{-1}}\right]_{j i} \operatorname{det}\left[1+e^{i \varphi_{m}} U(\sigma)\right],
$$

where $\varphi_{m} \equiv 2 \pi m / N_{s}\left(m=1, \ldots, N_{s}\right)$. As we will see in the next section, the presence of the Fourier sum increases the number of floating-point operations required for numerically stabilized calculations from $O\left(N_{s}^{3}\right)$ to $O\left(N_{s}^{4}\right)$ when the standard method from the grandcanonical ensemble is used.

For more details of the AFMC method and its practical applications, see Refs. [1, 3, 7 , 10, 11, 14-16, 19 22]. 


\section{NUMERICAL STABILIZATION}

In numerical AFMC calculations, the interval $[0, \beta]$ is divided into $N_{t}$ intervals of equal length $\Delta \beta=\beta / N_{t}$. The $N_{s} \times N_{s}$ matrix $U \equiv U(\sigma)$ in Eqs. (36) is then a time-ordered product

$$
U=U_{N_{\tau}} \cdots U_{1}
$$

of $N_{t}$ factors, where $U_{k} \equiv U\left(\sigma\left(\tau_{k}\right)\right)$ is the matrix representation in the single-particle space of the many-particle propagator $\hat{U}\left(\sigma\left(\tau_{k}\right)\right)$ for the $k$-th time slice. Each matrix $U_{k}$ has the form $U_{k}=e^{-\Delta \beta h_{k}}$, where $h_{k}$ is a complex, generally non-hermitian matrix generated from the stochastically selected fields $\sigma\left(\tau_{k}\right)$. In AFMC calculations, the product (7) is explicitly computed to obtain $U$.

At low temperature (i.e., large $\beta$ ), the number of factors in the product (7) becomes large. As the number of factors grows, the range of numerical scales represented in the product $U$ diverges, and the matrix $U$ becomes ill-conditioned, i.e., has large condition number [23] $\kappa(U) \equiv\|U\|\left\|U^{-1}\right\|$. This can make it impossible to extract information via Eqs. (55) and (6) about states in the interior of the single-particle spectrum, as the relevant energy scales are represented in $U$ only implicitly as the differences of much larger numbers [3, 21].

The known solution to this problem is to compute a decomposed form of $U$, such as a singular-value decomposition (SVD) or a QR decomposition [3, 24, 25]. These decompositions allow an accurate floating-point representation of $U$ by keeping the widely varying numerical scales in a separate diagonal matrix. Such a decomposition takes the form

$$
U=A D B=\left(\begin{array}{lll}
x & x & x \\
x & x & x \\
x & x & x
\end{array}\right)\left(\begin{array}{lll}
X & & \\
& X & \\
& & \\
& &
\end{array}\right)\left(\begin{array}{lll}
x & x & x \\
x & x & x \\
x & x & x
\end{array}\right),
$$

where $A$ and $B$ well-conditioned matrices (i.e., have condition numbers close to 1 ), and $D$ is diagonal with positive entries that represent the divergent scales contained in $U$. In Eq. (8) the size of the symbols indicates the respective magnitude of the matrix elements. In the SVD, we decompose $U=A D B$, where $A$ and $B$ are both unitary and the entries of $D$ are the singular values of $U$. To use a QR decomposition, we decompose $U=Q R$, where $Q$ is unitary and $R$ is upper triangular. We then set $A=Q$ and separate out the diagonal matrix $D$ from $R$ by scaling the rows of $R$ such that $R$ is either unit upper triangular [3] 


\section{STABILIZING CANONICAL-ENSEMBLE CALCULATIONS}

Once the multiplication in (7) is carried out stably and $U$ is available in a decomposed form $U=A D B$ (for a particular set of fields $\sigma$ ), it is necessary compute the partition function and the one-body densities. Canonical-ensemble calculations accomplish this using the Fourier sums (5) for the partition function and (6) for one-body observables. In this section, we first describe in Sec. IVA the method adapted from the grand-canonical calculations to canonical calculations [11], and then in Sec. IVB, we describe our improved method, which is the main subject of this paper.

\section{A. Standard method}

The grand-canonical partition function for a particular set of fields $\sigma$ is given by the determinant $\operatorname{det}(1+U)$ in Eq. (3) . Similar quantities appear in the canonical partition function (5) as $\operatorname{det}\left(1+e^{i \varphi_{m}} U\right)$, which must be computed for each value of $m=1, \ldots, N_{s}$. To compute these determinants from the decomposition $U=A D B$, one may factorize

$$
1+e^{i \varphi_{m}} A D B=A\left(A^{-1} B^{-1}+e^{i \varphi_{m}} D\right) B
$$

As discussed in Ref. [3], the addition of $e^{i \varphi_{m}} D$, which has widely diverging scales, to $A^{-1} B^{-1}$ does not introduce significant errors into the observables [26]. The quantity in parentheses can then be decomposed for each $m$ as $A^{-1} B^{-1}+e^{i \varphi_{m}} D=A_{m} D_{m} B_{m}$, so that

$$
\operatorname{det}\left(1+e^{i \varphi_{m}} A D B\right)=\operatorname{det} A \operatorname{det} A_{m} \operatorname{det} D_{m} \operatorname{det} B_{m} \operatorname{det} B
$$


The matrix decomposition is an $O\left(N_{s}^{3}\right)$ operation. Thus, the computation of the canonical partition (5) with this method is an $O\left(N_{s}^{4}\right)$ operation, since a matrix decomposition must be performed for each value of $m$ in the Fourier sum.

\section{B. Improved method}

If $U$ could be diagonalized by a similarity transformation, i.e., $U=P \Lambda P^{-1}$, where $P$ is invertible and $\Lambda_{i, j}=\delta_{i, j} \lambda_{i}$ is diagonal, then the eigenvalues $\lambda_{i}$ may be used to compute the determinant in $O\left(N_{s}\right)$ operations:

$$
\operatorname{det}\left(1+e^{i \varphi_{m}} P \Lambda P^{-1}\right)=\operatorname{det}\left(1+e^{i \varphi_{m}} \Lambda\right)=\prod_{k=1}^{N_{s}}\left(1+e^{i \varphi_{m}} \lambda_{k}\right)
$$

The calculation of the Fourier sum therefore becomes an $O\left(N_{s}^{2}\right)$ operation, requiring, however, a matrix diagonalization $\left[O\left(N_{s}^{3}\right)\right]$ beforehand. Thus such a method would overall requires $O\left(N_{s}^{3}\right)$ operations. A similar estimate applies to the calculation of the one-body densities.

However, the decomposition $U=A D B$ cannot simply be multiplied out to diagonalize $U$, as this would destroy information contained in all but the largest numerical scales in $D$. Instead, we can apply a simple transformation to stably diagonalize $U$. The equation we have to solve for an eigenvalue $\lambda$ of $U$ is

$$
A D B x=\lambda x .
$$

Multiplying both sides by $A^{-1}$ and defining $y=A^{-1} x$, we obtain

$$
D B A y=\lambda y,
$$

where $D B A$ is a row-stratified matrix, i.e., a well-conditioned matrix $B A$ multiplied on the left by a diagonal matrix $D$ whose entries vary widely in scale

$$
D B A=\left(\begin{array}{lll}
X & X & X \\
X & X & X \\
X & X & X
\end{array}\right) .
$$

The matrix $D B A$, although highly ill-conditioned, can be stably diagonalized by first balancing the matrix and then using the QR algorithm, as is done, e.g., in LAPACK [27]. We 
present numerical evidence of this in the next section. We can then stably determine the eigenvalues and eigenvectors of $U$. The eigenvalues of $U$ are exactly those of $D B A$, while the eigenvectors $x_{i}$ of $U$ can be obtained from the eigenvectors $y_{i}$ of $D B A$ by the transformation $x_{i}=A y_{i}$.

The Fourier sums (5) and (6) can easily be expressed in terms of the eigenvalues and eigenvectors of $U$, allowing one to stably compute observables from the decomposition $U=$ $A D B$ using $O\left(N_{s}^{3}\right)$ operations. For the partition function, we have

$$
\zeta_{N}(\sigma)=\frac{1}{N_{s}} \sum_{m=1}^{N_{s}} e^{-i \varphi_{m} N} \prod_{k=1}^{N_{s}}\left(1+e^{i \varphi_{m}} \lambda_{k}\right),
$$

while for the one-body densities, we compute

$$
\gamma_{k} \equiv \frac{1}{\zeta_{N}(\sigma) N_{s}} \sum_{m=1}^{N_{s}} e^{-i \varphi_{m} N}\left(\frac{1}{1+\lambda_{k}^{-1} e^{-i \varphi_{m}}}\right) \prod_{k=1}^{N_{s}}\left(1+e^{i \varphi_{m}} \lambda_{k}\right),
$$

so that (here the $j$-th column of the matrix $P$ is the eigenvector $x_{j}$ )

$$
\left\langle a_{i}^{\dagger} a_{j}\right\rangle_{N, \sigma}=\sum_{k} P_{j k} \gamma_{k} P_{k i}^{-1}
$$

We have compared the efficiency of the standard method and our improved method in the context of a particular many-body system that is of interest to cold atom physics. We consider two species of fermionic atoms (10 atoms of each type), moving in an isotropic three-dimensional harmonic trap and interacting with a contact interaction of zero range and infinite scattering length (known as the unitary limit) [10]. The single-particle basis (for a given species) consists of all eigenfunctions of the three-dimensional harmonic oscillator with at most $N_{\max }$ oscillator quanta. The number of single-particle states for this basis is given by $N_{s}=\left(N_{\max }+1\right)\left(N_{\max }+2\right)\left(N_{\max }+3\right) / 6$. In Fig. 1 we show the time to compute one sample in AFMC versus $N_{s}$ (the dimension of the matrices) for the standard method (open circles) and our improved method (solid circles). Our calculations were performed on a cluster with $2.8 \mathrm{GHz}$ Intel Xeon X5560 (Nehalem) CPUs. We utilized 128 cores for each simulation (i.e., for given values of $\beta$ and $\Delta \beta$ ). We observe that the new method yields a dramatic improvement in efficiency over the standard method. Many of our calculations in Ref. [10] were done for $N_{\max }=11$ oscillator quanta, for which the number of singleparticle states is $N_{s}=364$. In the standard method, these calculations would have been too time-consuming and thus impractical to carry out on current computers. 


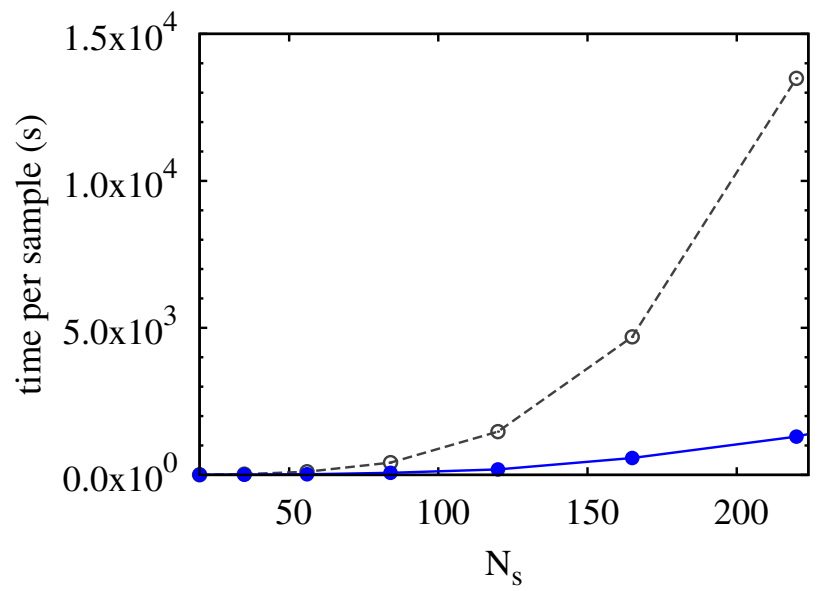

Figure 1. Comparing the timing of the standard and new methods for calculating observables from the stabilized propagator of Eq. (8). The time required to calculate one sample for a 20-atom cold Fermi gas at a temperature of $T=0.1 \hbar \omega$ ( $\omega$ is the trap frequency) and $\Delta \beta=1 / 32(\hbar \omega)^{-1}$ [10] is shown versus the number of single-particle states $N_{s}$. Open circles describe the standard method of Eqs. (9) and (10), while solid circles correspond to the new method using stabilized matrix diagonalization. Convergence for the condensate fraction in Ref. [10] was reached at $N_{\max }=11$ $\left(N_{s}=364\right)$, which would be impractical to compute using the standard method.

\section{STABILITY AND ACCURACY}

It is not obvious that the computation of the eigenvalues of the row-stratified matrix $D B A$ in Eq. (13) is numerically stable. In fact, the standard error analysis of the QR algorithm obtains a bound for the backward error which is proportional to the Frobenius norm of the matrix [28]. For our problem, this bound is many orders of magnitude larger than the relevant eigenvalues. This backward error also ignores the row-stratified structure of the original matrix.

The eigenvector problem of stratified (also known as graded) matrices has been explored previously; see, in particular, Ref. [29]. However, to our knowledge, no proof of the stability of the QR algorithm for stratified matrices is currently known.

This section has two parts. In Sec. VA, we first study the sensitivity of the eigenvalues and eigenvectors of the row-stratified matrix $D B A$ to small relative perturbations in its matrix elements. We find, using a modification of standard perturbation theory, that the problem is well-conditioned under essentially the same circumstances as for a matrix with a 
condition number close to 1.

In Sec. $\mathrm{VB}$, we test numerically the diagonalization of row-stratified matrices using LAPACK. We find, when the matrix is balanced beforehand, that the method is perfectly stable for matrices of the type considered here. We also demonstrate the accuracy of AFMC calculations with our improved method.

\section{A. Eigenvalue and eigenvector sensitivity}

Let $M$ be a row-stratified matrix

$$
M=D C=\left(\begin{array}{lll}
X & X & X \\
X & X & X \\
X & x & X
\end{array}\right),
$$

where $C$ is invertible and of unit scale (i.e., has eigenvalues of order 1 ) and $D=\operatorname{diag}\left\{d_{1}, \ldots, d_{n}\right\}$, where $d_{1} \geqslant d_{2} \geqslant \cdots \geqslant d_{n}>0$. (Here $M$ represents the matrix $D B A$ and $C$ represents the product $B A$ of Sec. IVB, Suppose also that $\lambda$ is a simple eigenvalue of $M$, and that $x$ and $y$ satisfy $M x=\lambda x$ and $y^{\dagger} M=\lambda y^{\dagger}$.

Standard perturbation theory [24] is concerned with the absolute sensitivity of $\lambda$ with respect to small perturbations in the entries of $M$. In particular, let $\delta M=\varepsilon E$ be a small perturbation of $M$ representing roundoff error in the entries of $M$. Here $\varepsilon$ is on the order of the machine precision and we may take $\|E\|_{2}=\|M\|_{2}$ (where $\|M\|_{2}=\max _{z \in \mathbb{C}^{N_{s}}}\|M z\|_{2} /\|z\|_{2}$ is the matrix 2-norm). Then the standard sensitivity analysis [24] gives

$$
|\dot{\lambda}(0)| \leqslant \frac{\|M\|_{2}}{s(\lambda)}
$$

where $\left.\dot{\lambda}(0) \equiv(d \lambda / d \varepsilon)\right|_{\varepsilon=0}$ and $s(\lambda) \equiv\left|y^{\dagger} x\right|$ is the condition of the eigenvalue $\lambda$. However, this result is not particularly useful for row-stratified matrices, since for the smaller eigenvalues, $\|M\|_{2}$ may be much larger than $\lambda$.

Fortunately, we can determine a bound for the relative error on $\lambda$ that exploits the structure of $M$. In particular, we find (see Appendix VI)

$$
|\dot{\lambda}(0) / \lambda| \leqslant \frac{\left\|C^{-1}\right\|_{2}}{s(\lambda)},
$$

which shows that the relative sensitivity of $\lambda$ does not depend on the condition number of $M$, but only on the condition of the matrix $C$ (which is of unit scale) and on the condition 
$s(\lambda)$ of the eigenvalue $\lambda$. We also note that, in the calculations described in this paper, $M$ is the product of matrix exponentials and therefore all of its eigenvalues are strictly nonzero, assuming a sufficient range in the floating-point representation.

In the SVD, $C$ is unitary, so $\left\|C^{-1}\right\|_{2}$ in Eq. (19) becomes 1 , and $|\dot{\lambda}(0) / \lambda| \leqslant 1 / s(\lambda)$. On the other hand, in the $\mathrm{QR}$ decomposition, $M=D R Q$, where $Q$ is unitary and $R$ is upper triangular. In this case $C=R Q$ and Eq. (19) becomes $|\dot{\lambda}(0) / \lambda| \leqslant\left\|R^{-1}\right\|_{2} / s(\lambda)$. In practice, the matrix $R$ is well-conditioned.

Thus, we conclude that the nonzero eigenvalues of the highly ill-conditioned but rowstratified matrix $M=D C$ are insensitive to roundoff error in $M$ when $s(\lambda)$ is not too small. This is similar to the situation for matrices with condition number close to 1, except that we have replaced the traditional analysis of the absolute error [24] with an analysis of the relative error.

A similar result can also be obtained for the eigenvector sensitivity. We find

$$
\left\|\dot{x}_{k}(0)\right\|_{2} \leqslant\left\|C^{-1}\right\|_{2} \sum_{i=1, i \neq k}^{N} \frac{\left|\lambda_{k}\right|}{\left|\lambda_{k}-\lambda_{i}\right|} \frac{1}{s\left(\lambda_{i}\right)},
$$

where $x_{k}$ is the $k$-th eigenvector and $\left.\dot{x}_{k}(0) \equiv\left(d x_{k} / d \varepsilon\right)\right|_{\varepsilon=0}$. Thus, the sensitivity of the $k$-th eigenvector depends on the fractional separation $\left|\lambda_{k}-\lambda_{i}\right| /\left|\lambda_{k}\right|$ of each eigenvalue from the target eigenvalue, as well as on the condition $s\left(\lambda_{i}\right)$ of each eigenvalue. Again, this result is similar to the usual result [24] a for well-conditioned matrix $M$, except that the absolute difference $\left|\lambda_{k}-\lambda_{i}\right|$ is replaced with the relative difference $\left|\lambda_{k}-\lambda_{i}\right| /\left|\lambda_{k}\right|$ multiplied by $\left\|C^{-1}\right\|_{2}$. We see that the eigenvector sensitivity depends only on the condition of the base matrix $C$ and of the individual eigenvalues, and not on the condition of $M$ itself.

\section{B. Numerical verification}

To test the stability of diagonalizing $D B A$, we computed the eigenvalues of a $N_{s} \times N_{s}$ complex matrix $U$ generated from a product of $N_{t}$ matrix exponentials

$$
U=e^{-\Delta \beta h} \cdots e^{-\Delta \beta h} \quad\left(N_{t} \text { times }\right),
$$

where $h$ is a randomly generated matrix (identical in each factor). The matrix $U$ is illconditioned and its calculation requires stabilized matrix multiplication. However, its eigenvalues and eigenvectors can be determined accurately from a single factor $e^{-\Delta \beta h}$, which is 
well-conditioned. Hence, such a matrix provides a convenient test for the diagonalization of matrices of the type that occur in AFMC.

For this test we chose the entries $h_{i j}$ to be complex numbers whose real and imaginary parts are randomly drawn from a uniform distribution on $(0,1)$. This type of matrix simulates the kind that occurs in the AFMC method. We computed $U$ in two different ways: (i) using unstabilized matrix multiplication (with the BLAS routine ZGEMM [30]), and (ii) using matrix multiplication stabilized with a QR decomposition. We then compared the eigenvalues and eigenvectors obtained by diagonalizing $U$ with those obtained by diagonalizing a single factor $e^{-\Delta \beta h}$. In exact arithmetic, the eigenvectors of $U$ should be identical to those of $e^{-\Delta \beta h}$, while the eigenvalues should be the $N_{t}$-th power of those of $e^{-\Delta \beta h}$.

We show in Fig. 2 the numerical relative error in the eigenvalues and eigenvectors of $U$ as a function of the condition number [31] of $U$, which grows monotonically with the number $N_{t}$ of factors. As the figure shows, the QR stabilization method together with diagonalization of $D B A$ is perfectly stable for products of the form (21).

To illustrate the accuracy of the AFMC calculations performed with the new stabilization method, we used again the example of the cold atom Fermi gas with contact interaction in a harmonic trap (for 20 particles). In Table \we show a comparison of the expectation values $\langle\hat{H}\rangle$ of the Hamiltonian using the standard and new stabilization methods, averaged over two samples. We also list the times required to compute these samples. The temperature used here $(T=0.1 \hbar \omega)$ is well within the region where stabilization is necessary. The results in Table I demonstrate that the two methods are numerically identical to a large number of digits. As a further test, we performed a full simulation with 4096 samples for $N_{\max }=6$ $\left(N_{s}=84\right)$ using both the new and standard methods. We obtained identical results for the two methods, with $\langle H\rangle=40.76528005 \pm 0.33 \hbar \omega$.

\section{CONCLUSION}

Numerically stabilized calculations of observables for a non-interacting propagator $\hat{U}$ are critical to performing AFMC calculations at low temperature and/or in large single-particle model spaces. We have described an improved method for computing the particle-numberprojected partition function and the expectation values of observables from a stabilized matrix decomposition $U=A D B$ of the propagator. The method works by employing a 
Figure 2. Relative errors in the eigenvalues and eigenvectors of a row-stratified, ill-conditioned matrix $U$ generated from a long product of matrix exponentials (21). The horizontal axis shows the approximate condition number of $U$ (computed as the magnitude of the ratio of the largest to smallest eigenvalues). On the vertical axis are the relative errors $\left|\lambda-\lambda_{\text {exact }}\right| /\left|\lambda_{\text {exact }}\right|$ and $\| v-$ $v_{\text {exact }}\|/\| v_{\text {exact }} \|$ of the eigenvalues and eigenvectors of $U$, respectively. Open triangles: eigenvalue errors from unstabilized matrix multiplication (using ZGEMM) and diagonalization; solid triangles: eigenvector errors from the same method. Solid circles: eigenvector errors from QR-stabilized matrix multiplication and diagonalization of $D R Q$; open circles: eigenvalue errors from the same method. We observe that for these matrices the QR stabilization method is perfectly stable up to condition numbers of $10^{300}$.

stabilized matrix diagonalization method for $U$ and computing the partition function and observables from the eigenvalues and eigenvectors of $U$. This new method reduces the $O\left(N_{s}^{4}\right)$ scaling of the standard method (when applied to the canonical ensemble) to $O\left(N_{s}^{3}\right)$ (where $N_{s}$ is the number of single-particle states). We have demonstrated that the new method can dramatically reduce the computational time of canonical AFMC calculations in the context of a trapped cold atom Fermi system. The method is also applicable to other physical systems such as nuclei, and can enable the study of systems in the canonical ensemble which previously could not be practically studied using AFMC.

The method relies on the stable diagonalization of an ill-conditioned but row-stratified matrix that arises as a product of matrix exponentials. We studied the perturbation theory for this problem and found that it is well-conditioned (i.e., insensitive to roundoff in the 
Table I. Energies and timings for two samples of a Monte Carlo simulation of a 20-atom, threedimensional isotropically trapped cold atomic Fermi gas at $T=0.1 \hbar \omega$. The atoms interact strongly with a contact interaction in the unitary limit of infinite scattering length. In the first and second columns (from the left) we list the maximal number $N_{\max }$ of oscillator quanta and the corresponding number $N_{s}$ of single-particle states. The third and fourth columns are, respectively, the expectation $\langle\hat{H}\rangle$ of the Hamiltonian averaged over two samples, and the time required to compute these two samples using the standard stabilization method. The fifth and sixth columns are the same quantities but using the new stabilization method. The numbers for $\langle\hat{H}\rangle$ shown here are not physical (as they are calculated from only two samples), but they clearly demonstrate that the two methods give numerically identical results. The times per sample for both methods are also shown in Fig. 1 ,

input matrix) under the same circumstances as for a well-conditioned matrix. Moreover, we demonstrated in numerical tests that the QR algorithm (as employed by LAPACK, in which it is preceded by matrix balancing) is numerically stable for this problem. This method may also apply to other calculations where information must be extracted from a dense, highly stratified matrix.

\section{ACKNOWLEDGEMENTS}

We thank G. W. Stewart for useful discussions. This work was supported in part by the Department of Energy grant DE-FG-0291-ER-40608. Computational cycles were provided 
by the NERSC high performance computing facility at LBL, and by the facilities of the Yale University Faculty of Arts and Sciences High Performance Computing Center. The acquisition of these facilities was partially funded by the National Science Foundation under grant No. CNS 08-21132.

\section{APPENDIX A}

To determine the relative sensitivity of the eigenvalues to roundoff error in the entries of $M$, we consider a perturbation of the form $\delta M=\varepsilon D F$, where $\|F\|_{2}=1$ (here $\|F\|_{2} \equiv \max _{z \neq 0}\|F z\|_{2} /\|z\|_{2}$ ) and $\varepsilon$ is small (on the order of the machine precision). Following Ref. [24], there exist differentiable $x(\varepsilon)$ and $\lambda(\varepsilon)$ in a neighborhood of $\varepsilon=0$ such that

$$
(M+\varepsilon D F) x(\varepsilon)=\lambda(\varepsilon) x(\varepsilon) .
$$

To determine the sensitivity of $\lambda$ with respect to the perturbation, we differentiate both sides with respect to $\varepsilon$ and set $\varepsilon=0$. If $y^{\dagger} M=\lambda y$ is the corresponding left eigenvector of $M$, It is easy then to see that

$$
|\dot{\lambda}(0)|=\frac{\left|y^{\dagger} D F x\right|}{\left|y^{\dagger} x\right|}=\frac{\left|y^{\dagger} D F x\right|}{s(\lambda)},
$$

where $x=x(0),\left.\dot{\lambda}(0) \equiv(d \lambda / d \varepsilon)\right|_{\varepsilon=0}$ and $s(\lambda) \equiv\left|y^{\dagger} x\right|$ is the condition of the eigenvalue $\lambda$. It then follows that

$$
\begin{aligned}
|\dot{\lambda}(0)| & =\frac{\left|y^{\dagger} D C C^{-1} F x\right|}{s(\lambda)} \\
& =\frac{|\lambda|\left|y^{\dagger} C^{-1} F x\right|}{s(\lambda)} .
\end{aligned}
$$

Using the fact that $\left\|y_{2}\right\|=\|x\|_{2}=1$ and $\|F\|_{2}=1$, we obtain

$$
|\dot{\lambda}(0) / \lambda| \leqslant \frac{\left\|C^{-1}\right\|_{2}}{s(\lambda)} .
$$

To obtain Eq. (20) we follow a similar procedure based on the analysis of Ref. [24].

[1] Y. Alhassid, A. Mukherjee, H. Nakada, and C. Özen,
Journal of Physics: Conference Series $\mathbf{4 0 3 , 0 1 2 0 1 2 ( 2 0 1 2 )}$.


[2] Y. Alhassid, in Fifty Years of Nuclear BCS, edited by R. A. Broglia and V. Zelvinsky (World Scientific, 2013) arXiv:1206.5834.

[3] E. Y. Loh Jr and J. E. Gubernatis, in Electronic phase transitions (Modern Problems in Condensed Matter Sciences), edited by W. Hanke and Y. Kopaev (North-Holland, 1992) pp. $177-235$.

[4] D. M. Charutz and D. Neuhauser, J. Chem. Phys. 102, 4495 (1995).

[5] S. Jacobi and R. Baer, J. Chem. Phys. 120, 43 (2004).

[6] A. Ohnishi, T. Ichihara, and T. Z. Nakano, in Proceedings of the 30th International Symposium on Lattice Field Theory, 2012, Cairns, Australia (2012).

[7] A. Bulgac, J. E. Drut, and P. Magierski, Phys. Rev. A 78, 023625 (2008).

[8] N. T. Zinner, K. Mølmer, C. Özen, D. J. Dean, and K. Langanke, Phys. Rev. A 80, 013613 (2009).

[9] J. Carlson, S. Gandolfi, K. E. Schmidt, and S. Zhang, Phys. Rev. A 84, 061602 (2011),

[10] C. N. Gilbreth and Y. Alhassid, Phys. Rev. A 88, 063643 (2013).

[11] Y. Alhassid, L. Fang, and H. Nakada, Phys. Rev. Lett. 101, 082501 (2008).

[12] J. Hubbard, Phys. Rev. Lett. 3, 77 (1959).

[13] R. Stratonovich, Dokl. Akad. Nauk. S.S.S.R. 115, 1097 (1957), [Sov. Phys. Dokl. 2 (1957) 416].

[14] Y. Alhassid, S. Liu, and H. Nakada, Phys. Rev. Lett. 99, 162504 (2007),

[15] H. Nakada and Y. Alhassid, Phys. Rev. C 78, 051304 (2008).

[16] H. Nakada and Y. Alhassid, Phys. Rev. Lett. 79, 2939 (1997).

[17] W. E. Ormand, D. J. Dean, C. W. Johnson, G. H. Lang, and S. E. Koonin, Phys. Rev. C 49, 1422 (1994).

[18] To make the Fourier sum numerically stable, a real chemical potential should be included, which we have omitted here for simplicity. For details, see Ref. [17].

[19] G. Lang, C. Johnson, S. Koonin, and W. Ormand, Phys. Rev. C 48, 1518 (1993).

[20] Y. Alhassid, D. J. Dean, S. E. Koonin, G. Lang, and W. E. Ormand, Phys. Rev. Lett. 72, 613 (1994).

[21] S. Koonin, D. Dean, and K. Langanke, Phys. Rep. 278, 2 (1997).

[22] Y. Alhassid, Int. J. Mod. Phys. B 15, 1447 (2001).

[23] Here the matrix norm $\|U\|$ is defined as $\|U\|=\max _{x \in \mathbb{C}^{N_{s}}}\|U x\| /\|x\|$. 
[24] G. H. Golub and C. F. Van Loan, Matrix computations, 2nd ed. (Johns Hopkins University Press, 1990).

[25] Z. Bai, C. Lee, R.-C. Li, and S. Xu, Linear Algebra Appl. 435, 659 (2011).

[26] This depends on the inclusion of a chemical potential to stabilize the Fourier sum [17], which we have omitted here for simplicity.

[27] E. Anderson, Z. Bai, C. Bischof, S. Blackford, J. Demmel, J. Dongarra, J. Du Croz, A. Greenbaum, S. Hammarling, A. McKenney, and D. Sorensen, LAPACK Users' Guide, 3rd ed. (Society for Industrial and Applied Mathematics, Philadelphia, PA, 1999).

[28] F. Tisseur, Backward stability of the QR algorithm, Tech. Rep. (Technical Report N 239, UMR 5585 Lyon Saint-Etienne, 1996).

[29] G. W. Stewart, On the Eigensystems of Graded Matrices, Tech. Rep. (Technical Report 200001, Institute for Advanced Computer Studies, University of Maryland, College Park, 2000).

[30] J. J. Dongarra, J. Du Croz, S. Hammarling, and I. S. Duff, ACM Trans. Math. Softw. 16, 1 (1990).

[31] In this test the condition number is estimated from $\left|\lambda_{\max } / \lambda_{\min }\right|$, i..e, the absolute value of the ratio of the largest-magnitude eigenvalue to the smallest-magnitude eigenvalue. 